\title{
Onicocriptosis en Pediatría: estudio clínico del tratamiento conservador
}

\section{Onicocriptosis in Pediatrics: clinical study of conservative treatment}

\author{
María Stoduto Charle ${ }^{1}$, Patricia Palomo López ${ }^{2}$ \\ ${ }^{1}$ Podologa. stodutopodologo@hotmail.com \\ ${ }^{2}$ Facultad de Podología, Universidad de Extremadura, Plasencia, España.patibiom@unex.es
}

Correspondencia:
María Stoduto Charle
Calle Puertas de Pro 46, Bajo
E-42002, Soria
Correo electrónico: stodutopodologo@hotmail.com

Fecha de recepción: 8 de octubre de 2013

Fecha de aceptación: 2 de febrero de 2014

Los autores declaran no tener ningún tipo de interés económico o comercial.

\section{RESUMEN}

La onicocriptosis es una afección de la uña muy común en consulta de pediatría ambulatoria, y en muchas ocasiones no sabe a quién derivar esta patología.

El diagnóstico es sencillo y clínico. La clínica de la onicocriptosis es eritema, edema y dolor a la presión de los rodetes periungueales. Si evoluciona presentará infección secundaria y posiblemente impotencia funcional para las actividades diarias y el deporte.

El tratamiento conservador y la espiculotomía tiene grandes beneficios por lo que se considera la técnica de elección en niños. Los casos clínicos que se presentan confirman una buena evolución de dichos tratamientos conservadores y espiculotomía, sólo recurriendo al tratamiento quirúrgico en onicocriptosis más evolucionadas.

La correcta higiene de los pies y un pronto diagnóstico nos permitiría realizar un tratamiento conservador evitando todos los riesgos que conlleva el tratamiento quirúrgico, y pudiendo sentir alivio instantáneo y una rápida vuelta a las actividades cotidianas.

Palabras clave: onicocriptosis; uña encarnada; espiculotomía; solución de burow; tratamiento conservador; niños.

\section{ABSTRACT}

The onicocriptosis is a condition of the nail very common in outpatient pediatric practice, and often do not know who derive this pathology.

The clinical diagnosis is simple. The clinic is onicocriptosis erythema, edema and pain on pressure Periungual impellers. If secondary infection evolves and possibly present functional disability for daily activities and sports.

Conservative treatment and espiculotomía has great benefits for what is considered the technique of choice in children. The clinical cases presented confirm a good performance of these conservative treatments and espiculotomía, only resorting to surgical treatment in more advanced onicocriptosis.

Proper foot hygiene and prompt diagnosis would allow us conservative treatment avoiding all the risks associated with surgery, and can feel instant relief and a rapid return to daily activities.

Key words: onichocryptosis; ingrown toenail; spiculectomy; burow's solution; conservative treatment; children.

Sumario: 1. Introducción. 2. Hipótesis. 3. Objetivos. 4. Material y Método. 5. Resultados. 6. Discusión. Bibliografía.

Referencia normalizada: Stoduto Charle, M., Palomo López, P. Onicocriptosis en Pediatría: estudio clínico del tratamiento conservador. Rev. Int. Cienc. Podol. 2014; 8(2): 83-89. 


\section{INTRODUCCIÓN}

La onicocriptosis es una patología del aparato ungueal en la que la lámina lesiona el rodete periungueal, por el contínuo traumatismo que provoca su crecimiento. Es una afección muy común, provoca dolor, inflamación y limitación funcional. En castellano, el término más empleado para referirse a ella es "uña encarnada"; y en inglés, "ingrown toenail". (Figura 1) 1 , $^{1,2}$

La onicocriptosis es el principal motivo de consulta por dolor ungueal 2,3,4,5,6,7,8,9,10,11,12,13 y puede aparecer en cualquier dedo, pero suelen aparecer principalmente en los primeros dedos de los pies ${ }^{1,3,9,12}$.

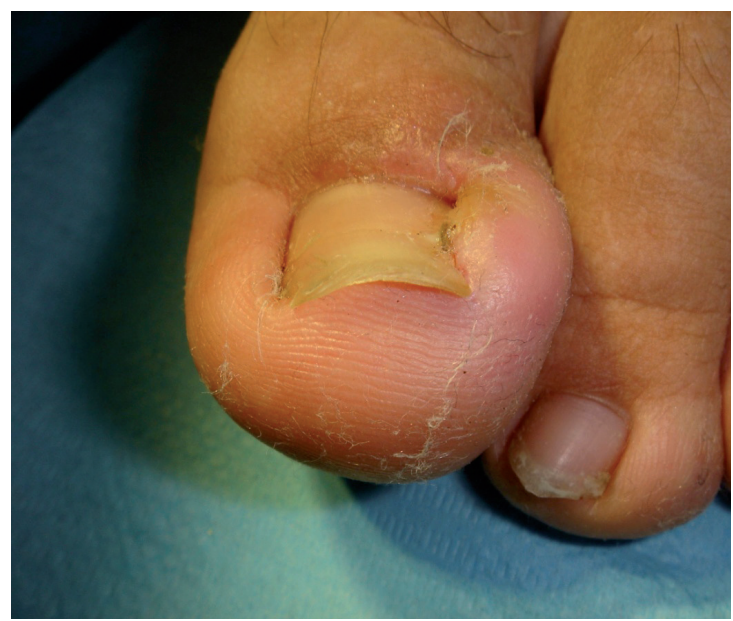

Figura 1. Onicocriptosis en varón de 10 años con rodete hipertrófico.

Esta afección es multifactorial, puede deber a factores extrínsecos como un traumatismo, factores mecánicos, un corte de la uña incorrecto $\mathrm{u}$ onicofagia (mordida o arrancamiento de la lámina ungueal), utilizar material para la higiene podal inadecuado, calzado inadecuado (Fig 2); o por factores intrínsecos como puede ser la hipersudoración, obesidad, diabetes, cabalgamiento del segundo dedo sobre el hallux y en algún caso puede que sea de forma congénita; etc. $1,2,3,5,6,9,10,11,12,13,14,15,16,17$

La onicocriptosis posee varios estadíos según su gravedad. En las primeras fases se produce un leve dolor debido al eritema y el edema en el rodete periungueal $1^{1,2,6,9,10,11,12,14,16,17}$. Si aumentan los síntomas y si además tiene espícula agravaría la alteración causando infección con secreción purulenta ya que ha sobrepasado levemente el pliegue ungueal. Si no se trata pronta y profesionalmente puede llegar a causar tejido de granulación y cronicidad, por lo que sólo se podría tratar con cirugía. (Figura 3) $1,2,3,6,9,10,11,12,13,14,16,17$

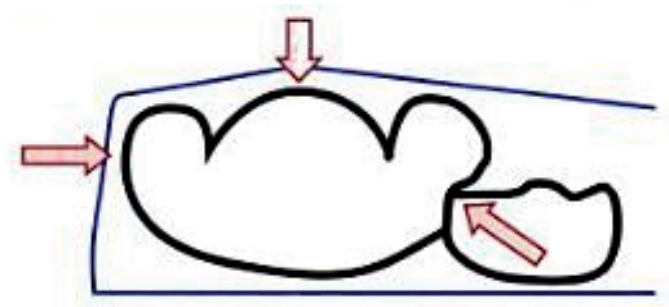

Figura 2. Posibilidad de onicocriptosis por roce del calzado ${ }^{1}$.

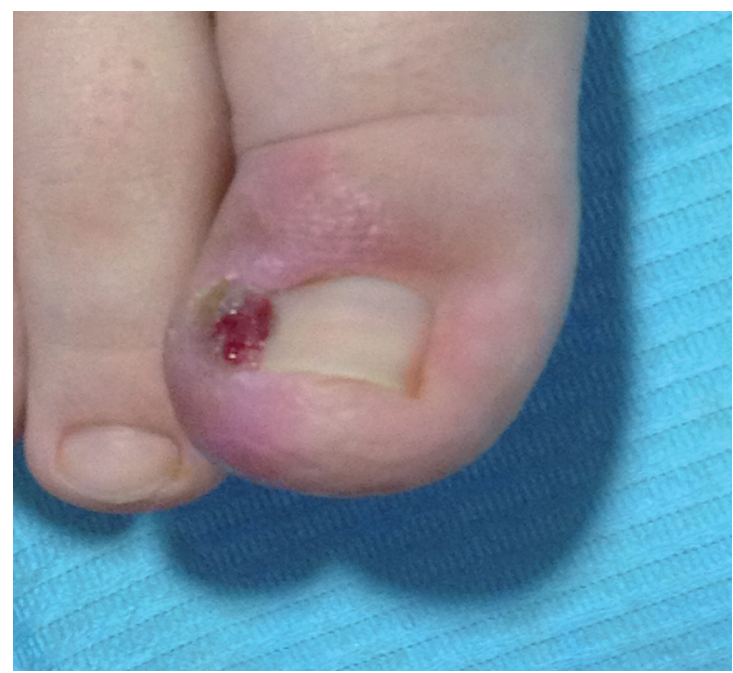

Figura 3. Onicocriptosis con tejido de granulación (varón 10 años).

Sin embargo, el tratamiento conservador, en los estadíos leves y/o con parte de granulación leve, nos permite erradicar, esta lesión sin recurrir a la cirugía en la mayoría de los ca$\operatorname{sos}^{3,5,9,10,11,12,13,18}$. Por lo que se puede decir que es un tratamiento ideal, en estadíos leves, para cualquier persona independientemente de la edad, pero con mayor interés para las edades pediátricas $^{1,2,3,6}$. 


\section{HIPÓTESIS}

La onicocriptosis es una alteración muy común en los niños ${ }^{4,5,7,8,13,14,16,17}$ y existen muchos tratamientos posibles ${ }^{1,3,11,12,13}$. La elección del tratamiento conservador y la espiculotomía en niños tiene gran éxito, y por ello predominaría antes que el tratamiento quirúrgico ${ }^{1,2,5,6}$, puesto que la cirugía puede presentar complicaciones por lo que debemos evitarla siempre que sea posible 10,12 .

\section{OBJETIVOS}

\section{General}

- Demostrar que en edades pediátricas, comprendida entre 3-10 años, el tratamiento conservador y la espiculotomía son el tratamiento de elección.

\section{Específicos}

- Identificar y analizar los principales problemas que causan la onicocriptosis.

- Enseñar a los pacientes una correcta cura doméstica de la afección.

- Enseñar a los pacientes una correcta higiene de los pies.

\section{MATERIAL Y MÉTODO}

\subsection{Tipo y duración del estudio}

El estudio realizado es de tipo descriptivo y desarrolla de forma puntual la técnica utilizada para el tratamiento de onicocriptosis en niños.

El trabajo de campo de éste estudio se ha desarrolló desde el inicio del curso académico 2012-2013 hasta el mes de Mayo de 2013, fecha de su conclusión.

\subsection{Material utilizado para el estudio}

Para realizar dicho proyecto de investigación se estudiaron 35 niños, todos ellos sin evidencias de traumatismo, ni afectos de patologías deformantes, sin intervenciones quirúrgicas, todos ellos pies pediátricos, con un rango de edad entre 3-10 años (desviación estándar: 6,5), de pacientes procedentes de la Clínica Universitaria de Podología de la Universidad de Extre- madura y la Clínica Podológica María Stoduto Charle, en Soria. En la muestra hay 24 niños y 11 niñas. Todos ellos acudieron a consulta de una forma privada, sin ser derivados. Se les informó del tratamiento que se realizaría dando así su consentimiento y la posibilidad de revocar ese consentimiento en cualquier periodo del tratamiento.

Para la realización del estudio y tratamiento, se prepararon varias cajas con material suficiente para poder ser utilizado convenientemente según el estadío de onicocriptosis de cada paciente. Las cajas de instrumental contenían:

- mangos de bisturí ${ }^{\circ} 3$.

- hojas de bisturí $n^{\circ} 15$.

- pinzas mosquito rectas.

- $\quad$ gubias $n^{\circ} 2$.

- tijeras rectas.

- alicates de corte recto.

- material desechable como: gasas, yodo líquido, pomada antibiótica, esparadrapo hipoalergénico.

- Solución de Burow modificada:

- Ácido acético 0,5\%.

- Ácido bórico 1\%.

- Solución acetotartrato de Aluminio $10 \%$.

- $\quad$ Agua destilada csp 250 cc.

Los tratamientos fueron fotografiados, para lo cual se utilizó el siguiente material:

- cámara reflex-digital Sony alfa 200.

- 1 trípode articulado.

- 1 tarjetas de memoria Compact-flash de 8 $\mathrm{Gb}$.

- $\quad$ se amplían las fotos a una escala de 1:4.

- para datos estadísticos se utiliza el Sofware SPSS, versión 15.

\subsection{Método de la Investigación}

La técnica utilizada en clínica para la onicocriptosis en niños es la espiculotomía y el tratamiento conservador de gasa, yodo y Solución de Burow.

La espiculotomía consiste en detectar si hay algún trozo de uña o espícula clavada en el rodete periungueal. Si la hubiese se realizaría con el bisturí del $\mathrm{n}^{0} 15$ un corte oblicuo ${ }^{1,2,3}$, es decir, en cuña siendo su vértice hacia la matriz. (Fig. 4) Siempre se debe cortar lo justo para elimi- 
nar la espícula sin alterar el lecho ungueal ni la matriz, dejando el borde lateral de la lámina ungueal sin ningún pico de uña ni zona cortante que pueda lesionar el rodete periungueal. Las curas se siguen en casa con pomada antibiótica en cura oclusiva por las mañanas y por la noche solución yodada.

La espiculotomía es un procedimiento simple, ambulatorio, indoloro, no requiere anestesia, tiene una rápida recuperación y desaparición inmediata del dolor, no tiene complicaciones y no necesita rehabilitación posterior, además de que no tiene recidivas ${ }^{2}$.

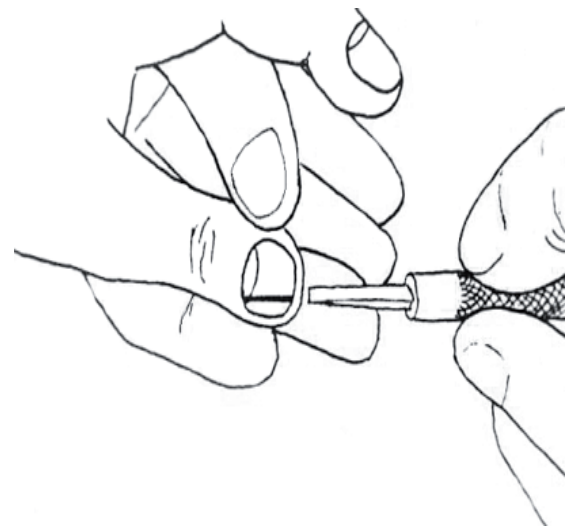

Figura 4: Técnica de espiculotomía².

Una vez eliminada la espícula o si no hubiese espícula, es decir, si símplemente hubiese una inflamación del rodete periungueal por fricción del crecimiento de la uña o anomalía del tamaño del rodete se realiza el tratamiento conservador de gasa y yodo ${ }^{1,3,11,12}$.

En el tratamiento conservador normalmente se utiliza algodón, pero debido a que ha presentado infecciones por quedar en la zona afectada hebras de algodón se decidió utilizar gasa. Cuando se retira la gasa para poner una nueva se extrae en su totalidad, no queda ninguna porción en el interior al contrario que el algodón.

Se realiza una mecha de gasa que se impregna con una gota de suero fisiológico para hacer más fácil su manipulación. Una vez mojada en suero se retuerce haciendo la forma de un único hilo un poco más grueso. Con la ayuda de una gúbia $\mathrm{n}^{\mathrm{0}} 2$ se introduce a lo largo de todo el canal periungueal afectado, intentando que quede debajo del borde de la lámina ungueal separándola del rodete periungueal ${ }^{1,3,12,13,16}$. El extremo de la mecha se introduce por el borde libre de la uña. No se debe dejar mucho sobrante de gasa ya que puede engancharse en los calcetines, y al ponerlos o quitarlos puede tirar del extremo haciendo que la gasa se salga de su recorrido.

Una vez que la gasa recorre y protege todo el recorrido del canal periungueal se empapa echando unas gotas de yodo. Se puede observar como la gasa queda de un color más oscuro debido a que está totalmente impregnada de yodo. (Figura 5) Esto permite una desinfección de toda la zona, y también seca la gasa endureciéndola y ofreciendo mayor resistencia en la acción de separación de las estructuras de la uña. Posteriormente se realiza una cura oclusiva con pomada antibiótica, gasa y esparadrapo, que debe permanecer cerrada durante 24 horas.

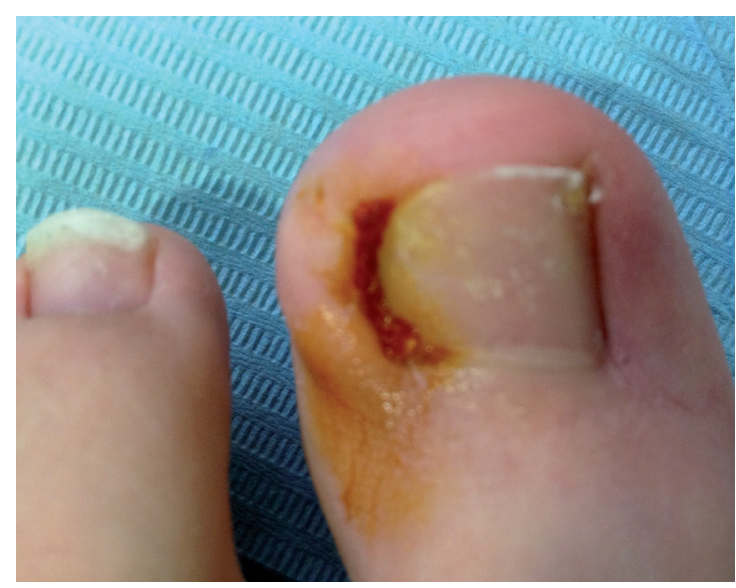

Figura 5. Tratamiento conservador de onicocriptosis con gasa y yodo en varón de 10 años.

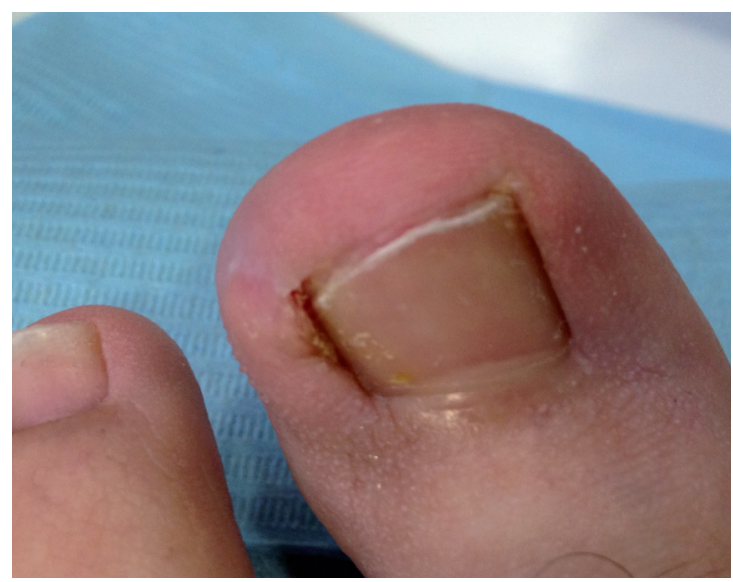

Figura 6: Evolución favorable de onicocriptosis en el caso del la Figura 5. 
Este tratamiento descrito lo realiza el podólogo una vez a la semana en consulta. El tratamiento que debe seguir en casa el paciente es diferente. Por la mañana debe lavarse con agua y jabón neutro el dedo para eliminar las impurezas que pueda haber, y se realiza una cura oclusiva con pomada antibiótica, gasa y esparadrapo; pero esta vez debe permanecer tapado hasta la noche cuando nos descalzamos, ya que debido a los agentes patógenos que hay en el calzado, hipersudoración, traumatismos inesperados, presión del calzado, etc. este vendaje oclusivo va a proteger la herida de todos ellos ${ }^{12}$. La pomada antibiótica ayuda a eliminar la infección y con ello la inflamación, pero al ser un producto húmedo puede macerar la zona $\mathrm{y}$ perjudicar el tratamiento reblandeciendo el rodete periungueal, lo que lo haría más vulnerable, o produciendo más infección baceriana o fungicida ${ }^{19,20,21}$. Para evitar esto, se debe lavar con agua y jabón nuevamente, impregnar una gasa con el preparado de Solución de Burow (fórmula magistral mejorada por los farmacéuticos Santiago Gómez Ortiz y Santiago Gómez Facundo, Barcelona) y aplicarla sobre toda la zona afectada durante 20-30 minutos dependiendo de la gravedad de la onicocriptosis. Incluso podría aplicar la Solución de Burow dos o tres veces al día ${ }^{1,19,20,21}$.

La Solución de Burow tiene buena tolerancia cutánea, y al poseer ácido acético inhibe el crecimiento de pseudomonas, al igual que el ácido bórico trabaja como antiséptico disminuyendo la infección. El Agua de Burow tiene un gran poder astringente, es decir que al disminuir la permeabilidad capilar de las células reduce edemas, exudados y congestión, por lo que reduce el dolor y la inflamación; también coagulan las proteínas sobre su superficie celular, es decir, realiza una dermoprotección y con ella efectos anhidróticos, evitando la sudoración de la zona; y por último al producir vasoconstricción de la zona en la que se aplica hace un ligero efecto hemostático ${ }^{19,20,21}$.

Una vez retirada la gasa con el preparado se vuelve a aplicar una gota de yodo sobre la gasa y la zona, eliminando el restante. Para que la piel pueda secarse de todos los productos y evitar que la zona se macere debe permanecer con el dedo sin tapar durante toda la noche, no se debe poner ningún vendaje, calcetín o similar hasta el día siguiente que se comenzaría el proceso otra vez.

Durante el periodo de estudio fuimos observando semana a semana las evoluciones individuales. No todos los casos se incluyeron en el mismo tiempo, ni evolucionaron y solventaron de igual manera.

La captación de casos fue difícil, ya que estas alteraciones normalmente acuden a consultas de atención primaria pediátrica o a cirugía principalmente, otros ni siquiera saben que tienen esta patología o la tratan en casa sin ningún consejo de un especialista ${ }^{6,16}$.

\section{RESULTADOS}

En 32 casos, de los cuales 32 eran niños y 11 niñas, la onicocriptosis se eliminó por completo. (Tabla 1) La infección se erradicó y con ello disminuyó la inflamación, por lo que había más espacio en el canal periungueal y la lámina ungueal disponía de mayor facilidad de crecimiento, evitando la fricción con el rodete periungueal.

\section{Número de casos de onicocriptosis}

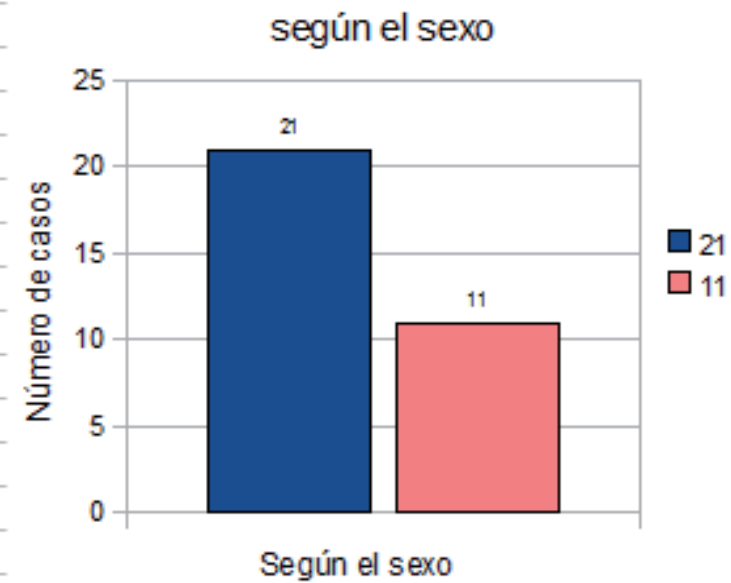

Tabla 1. Número de casos de tratamiento de onicocriptosis según el sexo.

Sólo en 3 casos no hubo los resultados esperados, no se eliminó la patología por completo. Pero disminuyó la inflamación y la infección 
pudiendo recurrir a la cirugía menor ambulatoria, en la que se utilizó la técnica de fenol, con menores complicaciones. (Tabla 2)

\section{Número de casos positivos y negativos}

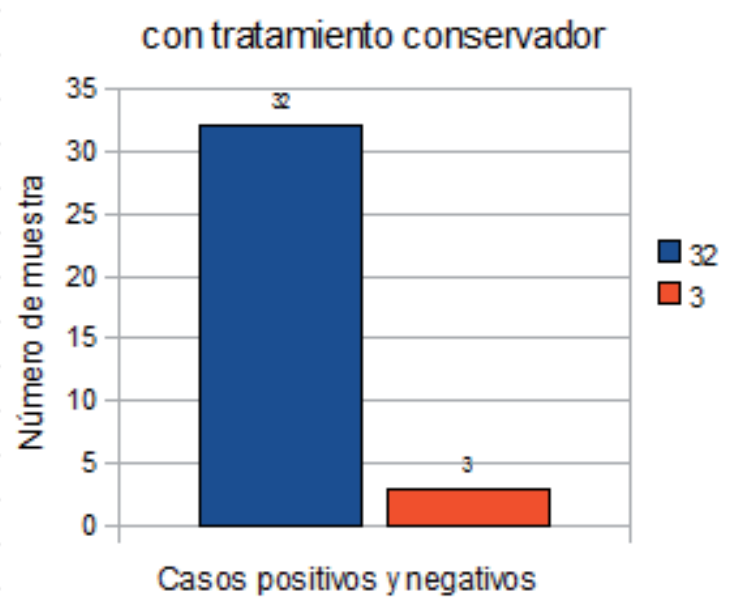

Tabla 2. Eficacia del tratamiento conservador, 32 casos positivos y 3 casos negativos en los que se tuvo que recurrir al tratamiento quirúrgico.

Se observó que la mecha de gasa se expulsaba con facilidad en algunos casos en los que los sujetos presentaban más de inflamación en la zona y mayor presión debido a un sobrepeso. Pero al realizar una cura semanal en consulta se solventó ese problema.

Destacó que la hipersudoración y el sobrepe-

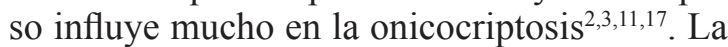
hipersudoración por la maceración e infección del rodete periungueal; y el sobrepeso por la presión que ejerce la lámina ungueal contra el rodete periungueal. Por lo que detectamos que los casos que presentasen estas alteraciones necesitan más tiempo para su total recuperación de la onicocriptosis.

\section{DISCUSIÓN}

El tratamiento fue positivo en la mayoría los casos. En los 3 casos en los que se tuvo que recurrir a la cirugía eran niños de 10 años, en los que destacaba un mayor grosor de la lámina ungueal, por lo que el tratamiento conservador no fue suficiente.

En este estudio de la onicocriptosis predominaron los niños ante las niñas, aunque esto depende mucho del tamaño de la muestra ya que en otros estudios predominan las mujeres. En un futuro habría que realizar el estudio en una muestra mayor e intentar averiguar el por qué se produce más frecuentemente en niños que en niñas.

La Solución de Burow modificada es de gran ayuda en esta patología, es una de las claves de este tratamiento ya que acorta el tiempo de curación $^{1,19,20,21}$. Se debería realizar estudiar la capacidad que tiene la Solución de Burow para acortar el periodo de tiempo de un tratamiento respecto a otro caso, del mismo grado de afección, en el que no se aplique dicha solución.

Debería prestarse mayor interés por enseñar a la población unas medidas de higiene de los pies adecuadas, mediante algún programa específico, independientemente de que tengan alguna alteración en dicha zona.

\section{BIBLIOGRAFÍA}

1. Martinez A. Podología. Podología: Atlas de Cirugía Ungueal. Buenos Aires; Madrid: Médica Panamericana, 2006. 27-38.

2. Gioseffi ML, Giachetti A, Sánchez L, De Freijo S, Sojo M. Onicocriptosis en pediatría: tratamiento conservador y espiculotomía. Arch Argent Pediatr. 2010;108(3):244-6.

3. Khunger N, Kandhari R. Ingrown toenails. Indian J Dermatol Venereol Leprol. 2012;78(3):279-89.

4. Islam S, Lin EM, Drongowski R, Teitelbaum DH, Coran AG, Geiger JD, Hirschl RB. The effect of phenol on ingrown toenail excision in children. J Pediatr Surg. 2005;40(1):290-2.

5. Mitchell S, Jackson CR, Wilson-Storey D. Surgical treatment of ingrown toenails in children: what is best practice?. Ann R Coll Surg Engl. 2011;93(2):99-102.

6. Kaleel SS, Iqbal S, Arbuthnot J, Lamont G. Surgical options in the management of ingrown toenails in paediatric age group. Foot. 2007;17(4):214-217.

7. Iglesias A, Tamayo L, Sosa-de-Martínez C, Durán-McKinster C, Orozco-Covarrubias L, Ruiz- 
Maldonado R. Prevalence and nature of nail alterations in pediatric patients. Pediatr Dermatol. 2001;18(2):107-9.

8. Chapeskie H, Kovac JR. Case Series: Soft-tissue nail-fold excision: a definitive treatment for ingrown toenails. Can J Surg. 2010;53(4):282-6.

9. Domingo H, Ribera M. Onicopatías más frecuentes en atención primaria. Jano. 2005;69(1.568):49-57.

10. Sardi JR. Onicocriptosis: (uña encarnada). Derm Venez. 1996;34(2):57-60.

11. Zavala K, Gutiérrez F, Bozalongo E. Manejo de la onicocriptosis en atención primaria. Caso clínico. Semergen. 2012;39(6):38-40.

12. Castro LM. Manejo de las uñas encarnadas en el $1^{\text {er }}$ nivel de atención. Rev Med Cos Cen. 2009;66(587):43-7.

13. Yang T, Yanchar NL, Lo AY, Jones SA. Treatment of ingrown toenails in the pediatric population. J Pediatr Surg. 2008;43(5):931-5.

14. Enriquez J, Alvarado A. Onicocriptosis en infantes. Reporte de 9 casos. Rev Cent Dermatol Pascua. 2004;13(3):168-71.

15. Kosaka M, Kusuhara H, Mochizuki Y, Mori H, Isogai N. Morphologic study of normal, ingrown, and pincer nails. Dermatol Surg. 2010;36(1):31-8.

16. Ikard RW. Onychocryptosis. J Am Coll Surg. 1998;187(1):96-102.

17. García FJ, Padín JM, Fernández D. Onicocriptosis crónica. Piel. 2005;20(1):53-5.

18. Rueff N, Gapany C. A rare ischemic complication of ingrowing toenail surgery in a child. Dermatol Surg. 2010;36(2):250-2.

19. Ballona R. Prurigo infantil. Folia Dermatol. Peru. 2008;19(3):149-53.

20. Novel V, et al. Podología: Guía Práctica. $2^{a}$ ed. Barcelona: Ed. Univers; 2008. 105-20.

21. Camean M, Buzo G, Rodriguez JC, Avila JR. Administración tópica y transdérmica. En: Santos B, Guerrero MD. Administración de medicamentos. Teoría y práctica. Madrid: Ed. Díaz de Santos; 1994. 75-98. 